\title{
A Somewhat Lower Frequency View of the Chandra Deep Field South
}

Jose Afonso ${ }^{*}{ }^{a b}$ Nissim Kanekar, ${ }^{c}$ Jayaram Chengalur, ${ }^{d}$ Hugo Messias, ${ }^{a b}$ Duncan Farrah, ${ }^{e f}$ and Bahram Mobasher ${ }^{g}$

a Observatório Astronómico de Lisboa/Faculdade de Ciências da Universidade de Lisboa Tapada da Ajuda, PT1349-018 Lisboa

${ }^{b}$ Centro de Astronomia e Astrofísica da Universidade de Lisboa

${ }^{c}$ National Radio Astronomy Observatory 1003 Lopezville Road, Socorro, NM 87801, USA

${ }^{d}$ National Centre for Radio Astrophysics

Ganeshkhind, Pune 411007, India

${ }^{e}$ Astronomy Centre, University of Sussex, Brighton, UK

${ }^{f}$ Astronomy Department, Cornell University

Ithaca, NY 14853, USA

${ }^{g}$ Department of Physics and Astronomy, University of California, Riverside, CA 92521, USA E-mail:jafonso@oal.ul.pt, nkanekar@aoc.nrao.edu, hmessias@oal.ul.pt, chengaluencra.tifr.res.in D.Farrahesussex.ac.uk, mobashereucr.edu

The Chandra Deep Field South (CDFS) is one of the most extensively observed regions of the sky, with some of the deepest multi-wavelength coverage ever: Chandra and XMM-Newton Xray observations, multi-band HST-ACS optical imaging and mid- to far-IR Spitzer observations, extensive deep ground-based imaging and spectroscopy with 8-m class telescopes such as the VLT and, finally, deep 1.4-GHz radio observations with the ATCA and the VLA. We are currently using the GMRT to image this field at $325 \mathrm{MHz}$ to very deep levels, which will allow us a first detailed glimpse of the nature of Ultra Steep Spectrum sources at the faintest radio flux levels.

Panoramic Radio Astronomy: Wide-field 1-2 GHz research on galaxy evolution

June 2-5 2009

Groningen, the Netherlands

\footnotetext{
* Speaker.
} 


\section{Introduction}

Understanding the formation and evolution of galaxies is one of the major challenges of modern astrophysics. Hierarchical models for structure formation developed over the last few years, achieving the high degree of predictive success that they do, are however still largely unconstrained, in particular in the way that light (galaxies) traces the underlying (dark) matter and how this relation evolves over time. The recent capabilities for performing extremely deep observations over a wide wavelength range can finally reveal the conditions in the distant, young Universe, avoiding biases ever present in any monochromatic view and allowing the study of different aspects of galaxy evolution (e.g., AGN activity, obscured and unobscured star-formation).

The Great Observatories Origins Deep Survey (GOODS) [1] was designed to unite the deepest observations from space- and ground-based facilities in two carefully selected fields, the Hubble Deep Field-North (HDF-N) and Chandra Deep Field-South (CDFS). With the deepest X-ray observations, using the Chandra X-Ray Observatory (CXO) and XMM-Newton, these areas have also been observed with the Spitzer Space Telescope at infrared wavelengths as part of several Legacy programmes. The Hubble Space Telescope (HST), using the Advanced Camera for Surveys (ACS), has also targetted these fields through a major allocation of time through HST Treasury programmes. The huge international effort to study these regions using also the most powerful ground-based instruments includes large spectroscopic programmes aiming to measure distances to most galaxies within the capabilities of present-day instrumentation, and to calibrate photometric redshifts for even fainter objects. The multitude of data available to the worldwide community currently makes these areas of the sky some of the most powerful to study galaxy formation and evolution.

Radio observations play a unique role in GOODS. Revealing the activity in a galaxy (either from star-formation or an AGN), radio emission is essentially unaffected by dust obscuration, which severely affects optical selection criteria [2], and is known to be an important source of bias at high redshift [3]. Radio emission is also a powerful probe of the highest redshift Universe: the most luminous radio galaxies, powered by an AGN, can in principle already be detected to the epoch of reionization with the current deep radio surveys ${ }^{1}$. Looking for such High Redshift Radio Galaxies (HzRGs) becomes even more attractive as they are associated with the most massive systems and are considered to identify the location of proto-cluster environments [5]. With large amounts of gas and dust and violent star formation [6] they are ideal targets to track the growth of Large Scale Structure in the Universe. The problem with the selection and study of such HighRedshift Radio Galaxies (HzRGs) relies not in the radio sensitivity, but often in that for the other wavelengths required to reveal the nature of such sources. Performing deep radio surveys in the GOODS fields can thus open up exciting new possibilities for the study of the highest-redshift Universe, through the identification and follow-up study of HzRGs.

The most efficient method to select HzRGs from radio surveys uses the observed correlation between radio spectrum steepness and redshift [7,8]. The reason behind such behaviour is still uncertain, but recent work suggests it can be linked to radio jets expanding in dense environments,

\footnotetext{
${ }^{1}$ As an example, source TNJ0924-2201, the highest redshift radio galaxy known ( $\left.z=5.19\right)$, has a radio $1.4 \mathrm{GHz}$ flux of 73 milliJansky [4], more than 1000 times the detection level currently reached in the deepest radio surveys.
} 
a situation one would more frequently find in proto-cluster environments in the distant Universe [9].

Only recently it has been possible to extend the search for USS sources to the microJansky regime, a fundamental step to find HzRGs at even higher distances and/or at earlier formation stages, when the radio power is still increasing.. This is mostly due to the success of recent low frequency facilities, as the Giant Metrewave Radio Telescope (GMRT), and it will be one of the main objectives behind the construction of future radio telescopes such as LOFAR. It is clear that the necessary follow-up studies for such searches will need the best possible multiwavelength data achievable, and even that may only be useful to find robust very high redshift candidates to be observed with future astronomical facilities.

\section{ATCA and GMRT observations of the CDFS}

We have selected one the the GOODS fields, the Chandra Deep Field South (CDFS), for deep multiwavelength radio observations suitable, among other objectives, to select faint USS sources. Coverage at $1.4 \mathrm{GHz}$ was performed with the Australia Telescope Compact Array (ATCA), reaching a sensitivity $(1 \sigma)$ of $\sim 14 \mu \mathrm{Jy}$, with a beam size of $\sim 17^{\prime \prime} \times 7^{\prime \prime}[10,11]$. These data have now been incorporated into the Australia Telescope Large Area Survey (ATLAS, see [12]).

Lower frequency $325 \mathrm{MHz}$ observations have been obtained by us with the Giant Metrewave Radio Telescope (GMRT). The observations, totaling around 50 hours of observing time, are in the final steps of processing. After the initial calibration, multifrequency synthesis cleaning and initial rounds of self-calibration, the sensitivity is at the $\sim 100 \mu \mathrm{Jy}$ level with a beam size of $15^{\prime \prime} \times 13^{\prime \prime}$. The resulting image has been used to perform a preliminary study on the USS sources in this field. Further work is on-going to improve the self-calibration procedure, remove residual sidelobes and increase the dynamic range, which should result in a significant improvement on the sensitivity.

\subsection{A sample of faint Ultra Steep Spectrum Sources in the CDFS}

In order to select a robust sample of USS sources in the CDFS we consider all radio detections within $30^{\prime}$ of the nominal center of the ATCA $1.4 \mathrm{GHz}$ radio image $\left(\alpha_{J 2000}=03^{\mathrm{h}} 32^{\mathrm{m}} 28^{\mathrm{s}}, \delta_{J 2000}=\right.$ $-27^{\circ} 48^{\prime} 30^{\prime \prime}$ ) [11]. This approximately corresponds to the central point of the GMRT observations ${ }^{2}$, thus ensuring the maximum sensitivity for the radio surveys considered. The catalogs at both radio frequencies were cross-correlated, and a sample of 28 sources with radio spectral index $\alpha_{325}^{1.4}<$ -1.3 (defined as $S \propto v^{\alpha}$ ) selected. After removal of sources near very strong detections, which may have the radio fluxes affected by the still incomplete $325 \mathrm{MHz}$ data processing, we assembled a robust sample of 18 USS sources in this area. The multiwavelength converage of this field can now be used to perform a first characterization of these sources.

\footnotetext{
${ }^{2}$ The aim point of the GMRT $325 \mathrm{MHz}$ observations was slightly shifted by $10^{\prime}$ due to the presence of a relatively strong radio source, PKS0326-278, with a $325 \mathrm{MHz}$ flux density $\sim 4.5 \mathrm{Jy}$, that would otherwise be at the edge of the primary beam; pointing errors throughout the observation would preclude the efficient sidelobe removal and limit the sensitivity.
} 


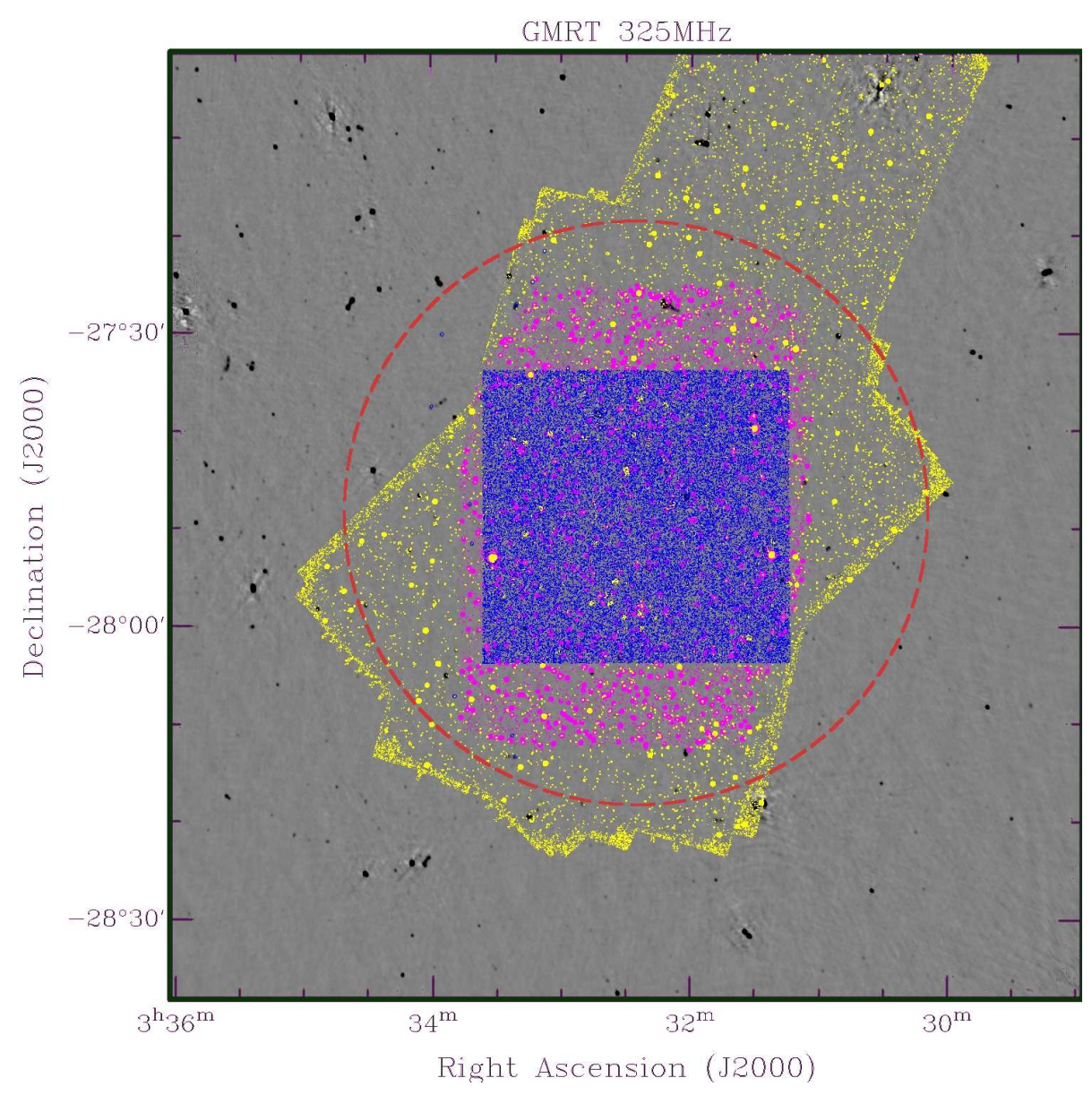

Figure 1: Central region of the current GMRT $325 \mathrm{MHz}$ image of the CDFS. The dashed circle denotes the region from where 18 USS sources were selected. GEMS sources are indicated in blue (the central square), those detected by SIMPLE in magenta (elongated rectangular region inscribed in the circle) and those by FIDEL in yellow (extending outside the circle).

\subsection{A first glimpse of CDFS USS sources at optical and infrared wavelengths}

Figure 11 shows the current radio image at $327 \mathrm{MHz}$ along with the area where USS sources were selected from and the coverage provided by other surveys in the optical (high resolution HST/ACS coverage by the program GEMS) and in the infrared (IRAC coverage by SIMPLE and MIPS coverage by FIDEL). The X-ray coverage provided by the Chandra 2 Ms observations [13] were also considered. The images and catalogs provided by these surveys were inspected for counterparts of the USS sources. In total, and due to the incomplete coverage of the radio region by the afore mentioned surveys, only two sources (out of five possible) have an optical counterpart in GEMS; a majority of sources show an infrared counterpart (10 out of 14 are detected with IRAC and/or MIPS) while only two (out of six) show an X-ray detection.

Four of the USS sources do not show any infrared counterpart. With radio fluxes from a few millijansky to above $40 \mathrm{mJy}$ at $1.4 \mathrm{GHz}$, these are Infrared Faint Radio Sources (IFRS, see [12]), 
sources whose nature is still unclear. Among the possible explanations are extremely redshifted AGN or extremely obscured galaxies invisible even in the mid-infrared. Two IFRS have recently been detected using VLBI $[14,15]$, implying that at least some of them do indeed have AGN cores. These are presumably so heavily obscured, or at such high redshift, that even Spitzer cannot detect their emission. This would also be consistent with the expected nature of many USS sources, as powerful AGN in dense environments at high redshift (see [9]).

The two USS sources with an optical identification also have a redshift determination from the COMBO-17 work [16] $(z=0.147$ and $z=0.662)$. The closer of the two is a rather curious object. The high resolution ACS optical image shows a face-on spiral galaxy (Figure 2). The SED of this source is typical of star formation (optical and mid-infrared) while the ultra-steep radio spectrum is more typical of AGN emission. However, at this redshift, the radio flux translates into a relativelly low luminosity $\left(L_{1.4 \mathrm{GHz}} \sim 10^{21.7} \mathrm{~W} \mathrm{~Hz}^{-1}\right.$, certainly not indicative of a powerful AGN. This behaviour might be related to the one observed in PRONGS (Powerful Radio Objects Nested in Galaxies with Star formation), unusual sources that have been observed in ATLAS [17] that also show a mixed appearance according to wavelength: an AGN according to the radio, while pure star formation is indicated by the optical and mid-infrared SED. Alternatively, the optical and mid-infrared identification may be erroneous, and corresponds to a nearby galaxy not related to the radio emission, from a background object.

\section{Conclusions}

GMRT $327 \mathrm{MHz}$ observations of the CDFS have been obtained and are in the process of processing. The final radio image currently reaches a sensitivity (rms) level of $\sim 100 \mu \mathrm{Jy}$. Together with the ATCA $1.4 \mathrm{GHz}$ coverage of the field, these data have been used to assemble a first robust sample of faint Ultra Steep Spectrum sources. The wide multiwavelength coverage of the CDFS will allow a detailed characterization of such sources, where the mid-infrared appears to have a fundamental value.

\section{References}

[1] M. Giavalisco, et al., The Great Observatories Origins Deep Survey: Initial Results from Optical and Near-Infrared Imaging, ApJL 2004 (600) L93

[2] Afonso, J., Hopkins, A., Mobasher, B., \& Almeida, C. Dependence of Dust Obscuration on Star Formation Rates in Galaxies, ApJ 2003 (597) 269

[3] Chapman, S. C., Smail, I., Blain, A. W., \& Ivison, R. J. A Population of Hot, Dusty Ultraluminous Galaxies at $z \sim 2$, ApJ 2004 (614) 671

[4] van Breugel, et al., A Radio Galaxy at $Z=5.19$, ApJL 1999 (518) L61

[5] Venemans, B. P., et al., Protoclusters associated with $z>2$ radio galaxies. I. Characteristics of high redshift protoclusters, A\&A 2007 (461) 823

[6] Reuland, M., Röttgering, H., van Breugel, W., \& De Breuck, C. Dust and star formation in distant radio galaxies, MNRAS 2004 (353) 377 


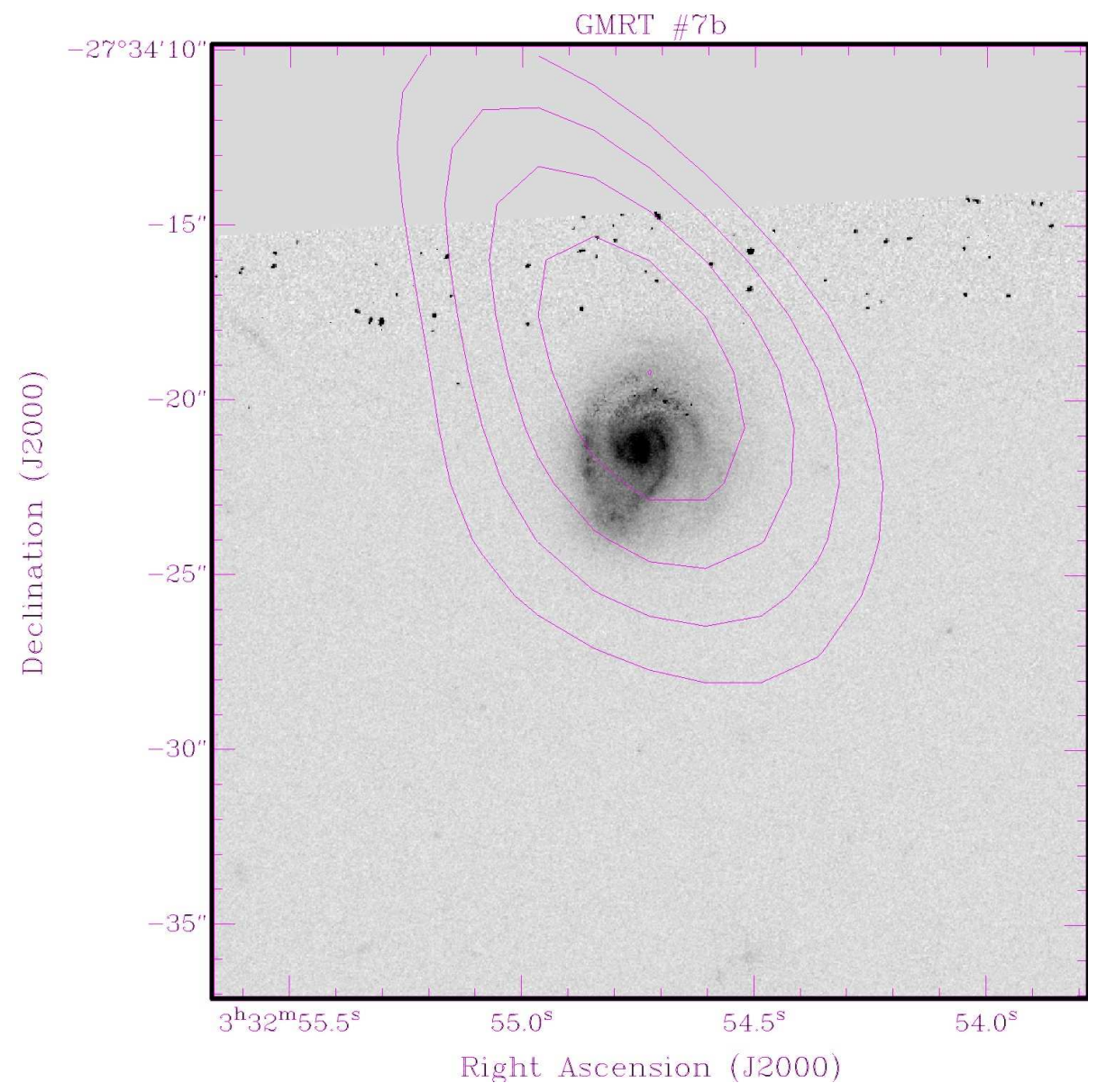

Figure 2: The closest of the selected USS, at a redshift of $z=0.147$, appears as a spiral galaxy in the optical image ( $z_{850}$ band, from the ACS coverage of this field by GEMS). With an optical and mid-infrared SED typical of star formation, and radio emission more indicative of an AGN, this radio source can have some relation to the unusual PRONGS.

[7] Miley, G. \& De Breuck, C. Distant radio galaxies and their environments, A\&ARv 2008 (15) 67

[8] De Breuck, C., van Breugel, W., Röttgering, H., \& Miley, G. A sample of 669 ultra steep spectrum radio sources to find high redshift radio galaxies, A\&AS 2000 (143) 303

[9] Klamer, I. J., et al., A search for distant radio galaxies from SUMSS and NVSS - III. Radio spectral energy distributions and the z- $\alpha$ correlation, MNRAS 2006 (371) 852

[10] Koekemoer, A., Mobasher, B., \& Norris, R. The ultra-deep $20 \mathrm{~cm}$ Australia telescope survey of the Chandra Deep Field South, NewAR 2003 (47) 391

[11] Afonso, J., et al., Optical and X-Ray Identification of Faint Radio Sources in the GOODS CDF-S Advanced Camera for Surveys Field, AJ 2006 (131) 1216

[12] Norris, R. P., et al., Deep ATLAS Radio Observations of the Chandra Deep Field-South/Spitzer Wide-Area Infrared Extragalactic Field, AJ 2006 (132) 2409

[13] Alexander, D., et al., The Chandra Deep Field North Survey. XIII. 2 Ms Point-Source Catalogs, AJ 2003 (126) 539 
[14] Norris R. P., et al., Very long baseline interferometry detection of an Infrared-Faint Radio Source, MNRAS 2007 (378) 1434

[15] Middelberg, E., et al., The first VLBI image of an infrared-faint radio source, AJ 2008 (491) 435.

[16] Wolf, C., et al., A catalogue of the Chandra Deep Field South with multi-colour classification and photometric redshifts from COMBO-17, A\&A 2004 (491) 913

[17] Norris, R., et al., arXiv:0804.3998 